\title{
Stress, Resilience and Moral Distress among Health Care Providers During COVID-19 Pandemic
}

\section{Ahmed Latif, ${ }^{1}$ Sobia Yaqub, ${ }^{2}$ Qudsia Anwar Dar, ${ }^{3}$ Umer Sultan Awan, ${ }^{4}$ Hina Farhat, ${ }^{5}$ Muhammad Abbas Khokhar ${ }^{6}$}

\begin{abstract}
Objective: This study aims to determine level of stress, resilience and moral distress among health care providers during covid-19 pandemic.

Methods: This is a cross-sectional study performed using an online questionnaire.

Data was collected from Health Care Providers, working in various tertiary care hospitals of Lahore, using an online questionnaire. Perceived stress scale (PSS), Connor-Davidson Resilience Scale (CD-RISC 10) and Moral Distress Thermometer were used to determine level of stress, resilience and moral stress respectively among the HCPs. Scores on the PSS can range from 0 to 40 with scores of $0-13,14-26$ and 27-40 being considered as low, moderate and high stress respectively. The Moral Distress Thermometer has scores ranging from 0 - 10 with value of $\geq 4$ considered high. Data was analyzed using SPSS version.23.Descriptive variables were reported as means and frequencies. Intergroup analysis was done using Chi square test with $\mathrm{p}<0.05$ taken as significant.

Results: A total of 278 ( $n=278)$ HCPs participated in study. According to the PSS (Perceived Stress Scale) scores, $5.03 \%$ (14) reported low, 86.69\% (241) moderate and 8.27\% (23) high stress levels. The mean stress score is $21.56+/-4.32$.

Providing patient care $($ mean $=2.28+/-1.15 \mathrm{SD})$ and transmitting infection to others (mean $=3.02+/-1.10 \mathrm{SD})$ were deemed major causes of stress. The mean CD-RISC score was $23.14+/-7.81$ SD. Only $10.8 \%(30)$ had a score of $\geq 32$. The mean Moral Distress score was 4.2+/-2.98 SD, with 53.2\% (149) participants reporting high Moral distress (score $\geq 4$ ).

Conclusion: The high level of stress among HCPs during COVID-19 pandemic highlights the need of urgent measures to overcome this psychological issue which ifleft un-addressed can affect performance of HCPs.

Key Words: Stress, Resilience, HCPs

How to cite: Latif A., Yaqub S., Dar A.Q., Awan S.U., Farhat Hina., Khokhar A.M., Stress, Resilience and Moral Distress among Health care Providers during COVID-19 pandemic. Esculapio 202 1;17 (01):79-82

DOI: https://doi.org/10.51273/esc21.2517116
\end{abstract}

\section{Introduction}

OVID-19 is an infectious disease of the respiratory tract caused by the SARS-COV-2. Originating in fish markets of Wuhan, China in the December

Ahmed Latif

3. Qudsia Anwar Dar

5. Hina Farhat

4. King Edward Medical University, Lahore

5. Department of Neurology, King Edward Medical University

Correspondence:

Dr. Ahmed Latif, Department of Oncology, King Edward Medical University

Email Id: sobia_yaqub@yahoo.com

Submission Date:

1st Revision Date:

Acceptance Date:

03-02-2021

12-02-2021

02-03-2021 of 2019, it has since become a global pandemic with more than 40 million cases and almost 1 million deaths. The common symptoms include fever, cough, shortness of breath, fatigue, new loss of sense of smell and taste and sometimes no symptoms at all. The disease mainly spreads via respiratory droplets among people who are in close proximity. ${ }^{1}$

HCPs (Health-Care Providers) typically face many stressors related to care for patients with different diseases. Especially in Pakistan, some of these include long working hours, high intensity shifts, non-availability of resources to provide the highest level of care and poor administration and management. Many of these lead to increased level of stress, anxiety, depre- 
ssion and lower levels of resilience among the HCPs. ${ }^{2}$ Although all HCPs face such challenges to some extent, their magnitude varies across different specialties. Recently, COVID-19 has been an additional source of stress and moral distress among the HCPs. From the fear of getting the infection to the stress of transmitting it to others, it has been a source of constant distress for the HCPs.

Stress, resilience and moral distress are the 3 parameters discussed in this study.

Stress can be defined as the degree to which you feel overwhelmed or unable to cope as a result of pressures that are unmanageable. ${ }^{3}$

Resilience is understood as referring to positive adaptation, or the ability to maintain or regain mental health, despite experiencing adversity. ${ }^{4}$

Moral distress is the painful psychological disequilibrium that results from recognizing the ethically appropriate action, yet not taking it, because of such obstacles as lack of time, supervisory reluctance, an inhibiting medical power structure, institution policy, or legal considerations. ${ }^{5}$

\section{Methods}

This is a cross-sectional study performed using an online questionnaire

Data was collected from Health care professionals (HCPs) working in various tertiary care hospitals of Lahore, using online questionnaire.

For stress, The Perceived Stress Scale (PSS) (Cohen, Kamarch, \& Mermelstein, 1983) has been used to assess the stress levels among the HCPs. ${ }^{6}$ To make the study easier for the participants, the recall-period has been shortened to 7 days. Scores on the PSS can range from 0 to 40 . Scores ranging from $0-13$ would be considered low stress. Scores ranging from 14-26 would be considered moderate stress. Scores ranging from 27-40 would be considered high perceived stress.

In addition, we have added seven items (stress due to work environment, patient care, personal safety, and indirect sources [home life, social isolation, other restrictions) with respect to the current COVID-19 pandemic. Each item is scored on a scale of 0-4, and mean stress scores were calculated.

For resilience, The Connor-Davidson Resilience Scale (Connor, K. M., \& Davidson, J. R., 2003) has been used. ${ }^{7}$ For our purposes, we have used the CD-
RISC 10 that includes 10 items. ${ }^{8}$ In the general population, a median score of 32 was determined (CampbellSills et al., 2009) with the lowest to highest quartiles being $0-29,30-32,33-36$, and 37-40.'

The Moral Distress Thermometer (Wocial, L.D. \& Weaver, M.T., 2013) has been used as the tool to assess moral distress among the HCPs (10). In a recent validation study, the threshold for high score was considered to be 4 (or higher).

This is a cross-sectional study performed using an online questionnaire. Our target population for this study were the HCPs working in multiple departments of different tertiary care hospitals in Pakistan. The questionnaire included the above-mentioned standard scales and assessment criteria. The data has been analyzed using SPSS ver. 26. Quantitative variables like age, resilience and moral distress scores have been described in terms of their mean values and ranges. Stress, resilience and moral distress scores have also been assessed in terms of percentages and frequencies and have been compared with variables like gender, age etc using chi square test $(\mathrm{p}=0.005)$.

\section{Results}

A total of $278(\mathrm{n}=278)$ participants took part in the study with ages ranging from 22 to 54 (mean age= 25.47+/-3.71 SD). 113 (40.6\%) were males and 165 $(59.4 \%)$ were females with majority of the subjects being single $(237 ; 85.3 \%)$. Out of the 278 participants, 265 (95.3\%) were practicing doctors while the remaining belonged to other fields. 101(36.33\%) belonged to Surgery and allied while the remaining 177 (63.66\%) belonged to Medicine and allied.

According to the PSS (Perceived Stress Scale) scores, $5.03 \%{ }^{14}$ of the participants reported low stress levels, $86.69 \%$ (241) reported moderate and $8.27 \%{ }^{23}$ reported high stress levels. The mean stress score is 21.56+/-4.32 SD. Moreover, providing patient care was more stressful (mean $=2.28+/-1.15 \mathrm{SD}$ ) as compared to stress that came from daily non-clinical work routine (mean $=2.18+/-1.09 \mathrm{SD}$ ). Similarly, transmitting the COVID-19 infection to others was deemed to be a greater cause of stress (mean $=3.02+/$ $1.1 \mathrm{SD})$ by the participants as compared to contracting the infection themselves (mean $=2.62+/-1.27 \mathrm{SD}$ ). Mean stress level from the need for social isolation was $2.55+/-1.44 \mathrm{SD}$ and mean stress from financial problems was $2.22+/-1.44 \mathrm{SD}$. 
Table 1:

\begin{tabular}{llcc}
\hline & Demographics & Count & Percentage \\
\hline \multirow{2}{*}{ Gender } & Total Participants & $\mathrm{n}=278$ & 100 \\
& Male & 113 & 40.6 \\
\multirow{2}{*}{ Marital Status } & Female & 165 & 59.4 \\
& Mangle & 237 & 85.3 \\
Profession & Doctor & 41 & 14.7 \\
\multirow{2}{*}{ Department } & Others & 265 & 95.3 \\
& Medicine and Allied & 177 & 4.7 \\
Age & Surgery and Allied & 101 & 63.66 \\
No. of family & \multicolumn{2}{c}{ 25.47+/-3.7 (range=54-22=32) } \\
members & \multicolumn{3}{c}{$5.87+/-1.87$ (range=16-2=14) } \\
\hline
\end{tabular}

Percentages of partigants with low. moderate and high. stress according vo PSS

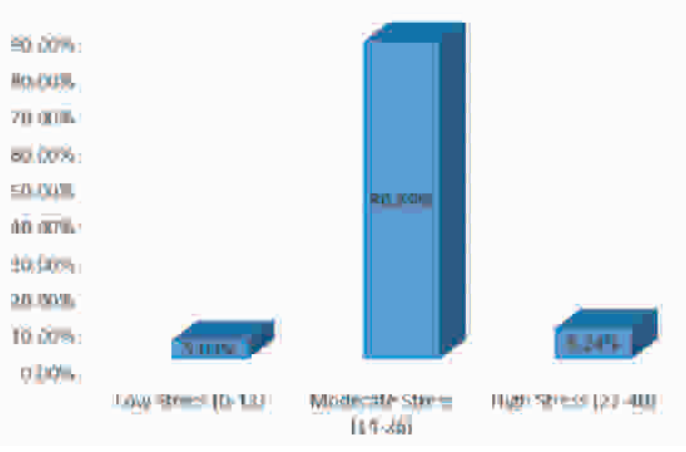

Mean Stress due to COVID-19 on a scale of 0.4

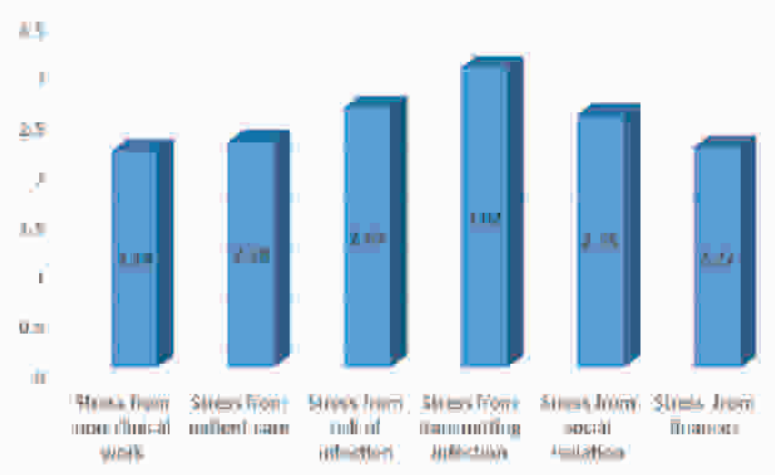

The mean CD-RISC (Connor Davidson Resilience Scale) score for all the participants was $23.14+/-7.81$ SD. Only $10.8 \%{ }^{30}$ of the participants had a CD-RISC score of 32 or above.

The mean Moral Distress score was 4.2+/-2.98 SD, with $53.2 \%$ (149) participants reporting high levels of Moral distress (score $\geq 4$ ).
Percentage of participants with HIgh and Law CU-BLSC Srare

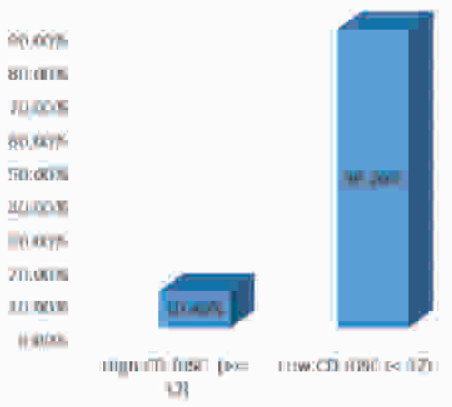

Percantage of Partiapants with high and low Moral Distres Störes.

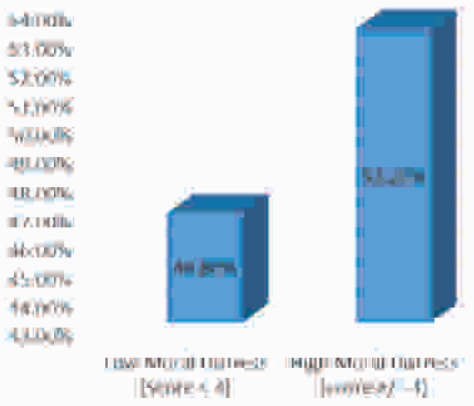

\section{Discussion}

COVID-19 has emerged as one of the biggest threats to mankind during the recent times. It has affected every aspect of human life. Public health has been its primary target.

One aspect of public health that is ignored most of the times is the issue of mental health. Healthcare services, by their nature, tend to be mentally and psychologically exhausting for the providers. Doctors, in general have lower levels of resilience in comparison to the normal population. Similarly, doctors of emergency medicine in particular, due to high workload and nature of their work, are more prone to burnout as compared to other specialties of medicine. ${ }^{11}$

During these hard times of COVID-19, when there is air of fear and uncertainty, the psychological and mental health of the individuals has been more severely affected. There is increased stress, depression and anxiety, even among the general population. ${ }^{12}$ Health Care Providers are the first line of defense against this pandemic. Naturally, they are the ones that are the most susceptible to its hazardous effects, both physically and psychologically. ${ }^{13}$

Previous literature suggests that the HCPs treating patients with COVID-19 reported higher levels of anxiety and low self-efficacy levels. ${ }^{14,15}$ Other studies on Wuhan medical professionals reported greater 
susceptibility to stress, anxiety, and depression, suggesting that the mental health of the frontline HCPs should be closely examined. ${ }^{16}$ In our study we have reported self-perceived levels of stress, resilience and moral distress among the HCPs. In this survey, the participants reported increased levels of stress, lower levels of resilience and high levels of moral distress. All of these findings correlate with each other. This means that higher levels of stress are associated with lower levels of resilience and higher levels of moral distress. The findings of our study are consistent with the previously mentioned studies.

It was interesting to note that more participants feared transmitting the infection to others rather than getting themselves infected. This may be due to the fact that people tend to show pro-social behavior under stressful conditions. ${ }^{17}$

There are certain limitations to this study. First one is the small sample size. Another one is the biased sampling that involves most participants between the ages of 20 and 30 years. Thirdly, the online data collection technique in itself has many flaws including generalized responses. ${ }^{18}$

\section{Conclusion}

The mental and psychological effects of the current COVID-19 pandemic are widespread. The HCPs face these effects head on. In addition to providing PPE to them, proper steps should be taken to preserve the mental health of our HCPs. Right actions taken timely will result in the provision of better healthcare to our patients, which is the basic aim of a sound health system.

\section{Conflict of Interest: $\quad$ None}

\section{References}

1. Coronavirus disease (COVID-19) [Internet]. Who. int. 2020 [cited 22 October 2020]. Available from: https://www.who.int/emergencies/diseases/novelcoronavirus-2019/question-and-answers-hub/q-adetail/coronavirus-disease-covid-19

2. McKinley N, McCain RS, Convie L, Clarke M, Dempster M, Campbell WJ, Kirk SJ. Resilience, burnout and coping mechanisms in UK doctors: a cross-sectional study. BMJ open. 2020 Jan 1;10(1).

3. Stress [Internet]. Mental Health Foundation. 2020 [cited 17 September 2020]. Available from: https:// www.mentalhealth.org.uk/a-to-z/s/stress\#: : text= Stress $\% 20$ can $\% 20$ be $\% 20$ defined $\% 20$ as,of $\% 20$ press ures $\% 20$ that $\% 20$ are $\% 20$ unmanageable.

4. Herrman H, Stewart DE, Diaz-Granados N, Berger EL, Jackson B, Yuen T. What is resilience?. The Canadian Journal of Psychiatry. 2011 May; 56(5): 258-65.

5. Corley MC, Elswick RK, Gorman M, Clor T. Development and evaluation of a moral distress scale. Journal of advanced nursing. 2001 Jan 26;33(2):250-6.

6. Cohen, S., Kamarck, T., \& Mermelstein, R.. A global measure of perceived stress. J Health Soc Behav. 1983; 24(4): 385-396.

7. Connor, K. M., \& Davidson, J. R.. Development of a new resilience scale: the Connor Davidson Resilience Scale (CD-RISC). Depress Anxiety. 2003; 18(2): 7682. doi:10.1002/da.10113

8. Campbell-Sills, L., \& Stein, M. B.. Psychometric analysis and refinement of the Connor-davidson Resilience Scale (CD-RISC): Validation of a 10-item measure of resilience. J Trauma Stress. 2007; 20(6): 1019-1028. doi:10.1002/jts.20271

9. Campbell-Sills, L., Forde, D. R., \& Stein, M. B.. Demographic and childhood environmental predictors of resilience in a community sample. J Psychiatr Res. 2009; 43(12): 1007-1012. doi:10.1016/j.jpsychires. 2009.01.013.

10. Wocial, L. D., \& Weaver, M. T.. Development and psychometric testing of a new tool for detecting moral distress: the Moral Distress Thermometer. J Adv Nurs. 2013; 69(1): 167-174. doi:10.1111/j.13652648.2012.06036.x

11. Barzilay R, Moore TM, Greenberg DM, DiDomenico GE, Brown LA, White LK, Gur RC, Gur RE. Resilience, COVID-19-related stress, anxiety and depression during the pandemic in a large population enriched for healthcare providers. Translational psychiatry. 2020 Aug 20;10(1):1-8.

12. Buchanan TW, Preston SD. Stress leads to prosocial action in immediate need situations. Frontiers in Behavioral Neuroscience. 2014 Jan 22;8:5.

13. Behrend TS, Sharek DJ, Meade AW, Wiebe EN. The viability of crowd sourcing for survey research. Behavior research methods. 2011 Sep 1;43(3):800.

\section{Authors Contribution}

MAK,SY: Conceptionlization of Project

AL,USA: Data Collection

AL,SY: Literature Search

QAD,HF: Statistical Analysis

SY,MAK: Drafting, Revision

SY,MAK: Writing of Manuscript 